\title{
Photochemical tagging for rapid quantitation of unsaturated fatty acids by mass spectrometry
}

Xiaoxiao Ma, ${ }^{[a],[b]}$ Xu Zhao, ${ }^{[a][c]} J u n j i e ~ L i,{ }^{[b]}$ Wenpeng Zhang, ${ }^{[b]} \mathrm{Ji}-$ Xin Cheng, ${ }^{[b][d]}$ Zheng Ouyang*, ${ }^{[b]}$ Yu Xia*[a]

[a] Dr. X. Ma, X. Zhao, Prof. Y. Xia,

Department of Chemistry, Purdue University, West Lafayette, IN 47907 (USA)

E-mail: yxia@purdue.edu

[b] Dr. X. Ma, Dr. J. Li, Dr. W. Zhang, Prof. J.-X. Cheng, Prof. Z. Ouyang

Weldon School of Biomedical Engineering, Purdue University, West Lafayette, IN 47907 (USA)

E-mail: ouyang@purdue.edu

[c] Current address: Department of Chemistry, Tsinghua University, Beijing, 100084 (China)

[d] Purdue University Center for Cancer Research, Purdue University, West Lafayette, 47907

Correspondence should be addressed to Y. X. (yxia@purdue.edu) or Z. O. (ouyang@purdue.edu). 


\section{Chemicals and Materials}

All FAs and deuterium-labeled FA standards were purchased from Cayman Chemical (Ann Arbor, MI) and used without further purification. Pooled human plasma (Li Heparin as anticoagulant) was purchased from Innovative Research (Novi, MI). Other chemicals were purchased from Sigma Aldrich (St. Louis, MO).

\section{Cell culture}

All cells were cultured in $37{ }^{\circ} \mathrm{C}$ moisture incubator with $5 \% \mathrm{CO}_{2}$ supply. Prostate cancer PC 3 cells were maintained in F-12K medium supplemented with 10\% fetal bovine serum (FBS), 100 units/mL penicillin and $100 \mu \mathrm{g} / \mathrm{mL}$ streptomycin. Normal prostate epithelial RWPE1 cells were cultured in Keratinocyte Serum Free Medium supplemented with $30 \mu \mathrm{g} / \mathrm{ml}$ bovine pituitary extract and $0.2 \mathrm{ng} / \mathrm{ml}$ human recombinant epidermal growth factor (Invitrogen, Carlsbad, CA).

\section{Protocols for the extraction of FAs from human plasma and cells}

\subsection{Extraction of FAs from human plasma}

1. Add $30 \mu \mathrm{L}$ dPBS to $20 \mu \mathrm{L}$ human plasma in a $16 \mathrm{~mm} \times 125 \mathrm{~mm}$ glass tube, followed by addition of $60 \mu \mathrm{L}$ methanol. The mixture was then acidified with $1 \mathrm{M} \mathrm{HCl}$ to reach a final concentration of $25 \mathrm{mM}$.

2. After the addition of $0.1 \mathrm{~mL}$ isooctane, the sample is vortexed and centrifuged at $3000 \mathrm{~g}$ for 1 minute to separate layers. The top layer is removed and transferred to a $10 \mathrm{~mm} \times 75$ mm glass tube.

3. Repeat step 2 for one time.

4. Combine the organic layers. Dry down the extract under vacuum or using nitrogen flow.

\subsection{Extraction of FAs from cells:}

1. Five million cells in $1 \mathrm{~mL}$ PBS in a $16 \mathrm{~mm} \times 125 \mathrm{~mm}$ glass tube was centrifuged for 2 minutes at $3000 \mathrm{rpm}$, after which the upper aqueous layer was discarded.

2. Into the cells was added $300 \mu \mathrm{L}$ dPBS, followed by addition of $600 \mu \mathrm{L}$ methanol. The cell suspension was acidified with $\mathrm{HCl}$ to reach a final concentration of $25 \mathrm{mM}$

3. After the addition of $1 \mathrm{~mL}$ isooctane (2,2,4-trimethylpentane), the sample was vortexed and centrifuged at $3000 \mathrm{~g}$ for 1 minute to separate layers. The top layer was removed and transferred to a $10 \mathrm{~mm} \times 75 \mathrm{~mm}$ glass tube.

4. Repeat step 3. 
5. Combine the organic layers. Dry down the extract under vacuum or nitrogen flow.

\section{Photochemical tagging and tandem MS analysis}

For quantitation, MUFAs were dissolved in 5\% ethanol in acetone/water $(50 / 50 \mathrm{v} / \mathrm{v})$ prior to MS analysis, whereas PUFAs were dissolved in $40 \%$ ethanol in acetone/water $(50 / 50 \mathrm{v} / \mathrm{v})$. To facilitate FA detection by negative mode nanoESI-MS, $0.5 \%$ (v/v) $\mathrm{NH}_{4} \mathrm{OH}\left(28 \%-30 \%\right.$ as $\left.\mathrm{NH}_{3}\right)$ was added into all FA solutions. NanoESI tips of $\sim 10 \mu \mathrm{m}$ outer diameter were pulled using borosilicate glass capillary tips $(1.5 \mathrm{~mm}$ o.d. and $0.86 \mathrm{~mm}$ i.d.) by a P-1000 Flaming/Brown micropipette puller (Sutter Instrument, Novato, CA, USA). Lipid solution was loaded from the back opening of the borosilicate glass tip. A stainless steel wire was inserted to the tip to serve as the electric contact, with the nanoESI tip aligned with the MS sampling orifice. To initiate photochemical tagging via PB reactions ${ }^{1}$, A low-pressure mercury (LP-Hg) lamp (254 nm, Model No.: 80-1057-01, BHK, Inc., CA, USA) was placed $1.0 \mathrm{~cm}$ from the nanoESI emitter. All MS experiments were performed on a 4000 QTRAP triple quadrupole/linear ion trap (LIT) hybrid mass spectrometer (Applied Biosystems/Sciex, Toronto, Canada), and its schematics is shown in Figure S1. The instrument parameters were as follows: ESI voltage, -1200-1800V; curtain gas, $10 \mathrm{psi}$; interface heater temperature, $40^{\circ} \mathrm{C}$; declustering potential: $-20 \mathrm{~V}$. For MS/MS analysis of selected PB reaction products, the isolation width was set to $1.5 \mathrm{Th}$, and the precursor intensity was kept at around $4 \times 10^{6}$ counts. The ion injection time was 10-200 ms. The collision energy (CE) used for PB reaction products of FAs was optimized to be $35 \mathrm{~V}$ (beam-type CID) or 50 a.u. (resonance trap CID). For neutral loss scan (NLS), a CE of $35 \mathrm{~V}$ was used.

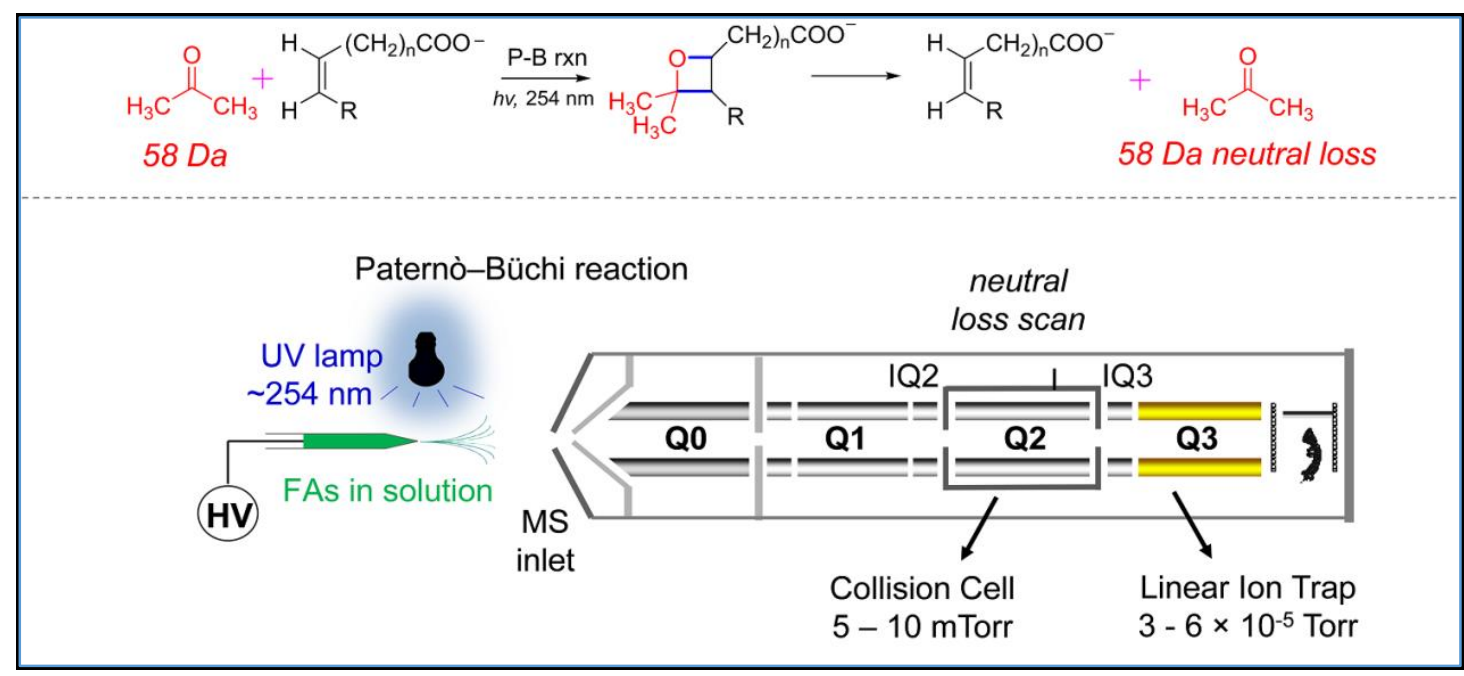

Scheme S1. (top) The reaction scheme of PB reaction and retro-PB reactions during MS/MS. (bottom) The experimental setup coupling PB reaction with nanoESI-MS/MS on an AB Sciex Qtrap 4000 MS for the quantitation of unsaturated FAs by NLS. 


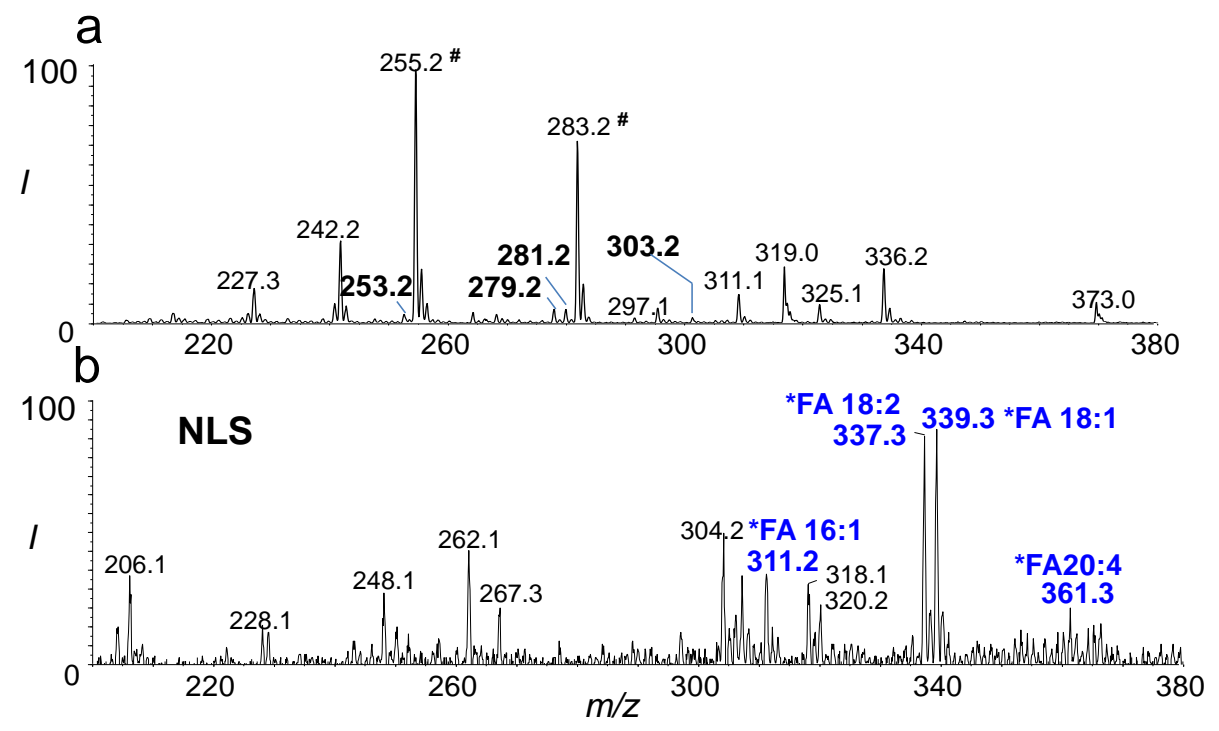

Figure S1. Negative ion mode nanoESI mass spectra of FA extract from $2 \mu \mathrm{L}$ rat blood after 25 times diultion: (a) before tagging and (b) NLS (58 Da) after tagging. The most abundant peaks in the MS spectrum (a) were in fact background chemical interferences, annotated with “"””.

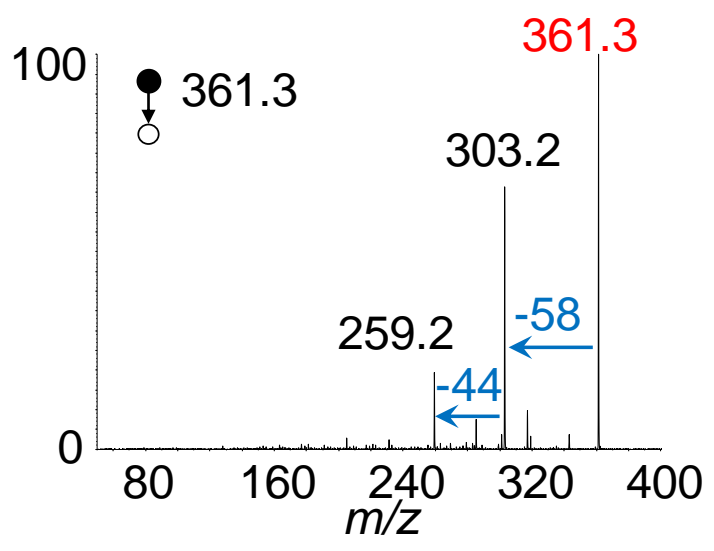

Figure S2. CID spectrum of PB products of FA 20:4 at $\mathrm{m} / \mathrm{z} 361.3$, where the dominant fragmentation channels are the neutral losses of $58 \mathrm{Da}$ (acetone) and $44 \mathrm{Da}\left(\mathrm{CO}_{2}\right)$. 
FA 18:1(11Z)

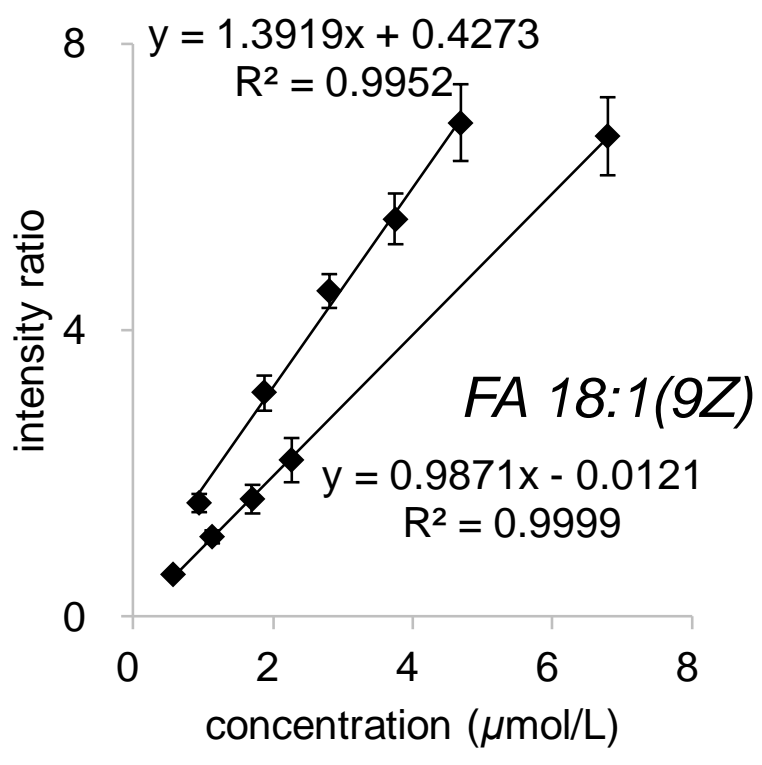

Figure S3. Calibration curves for pure oleic acid (FA 18:1 (9Z)) and cis-vaccenic acid (FA 18:1 (11Z)) based on 58 Da NLS. Note that these two calibration curves do not overlay with each other because tagged products of each isomer show different degrees of tag loss.

\section{Analysis of free FAs in human plasma}

5.1 Estimation of FA extraction efficiency (using isotope-labelled internal standards)

human plasma

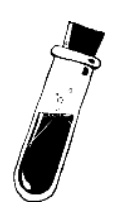

add IS into human plasma

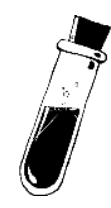

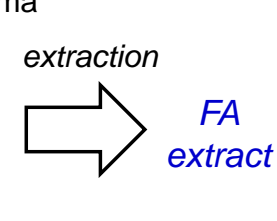
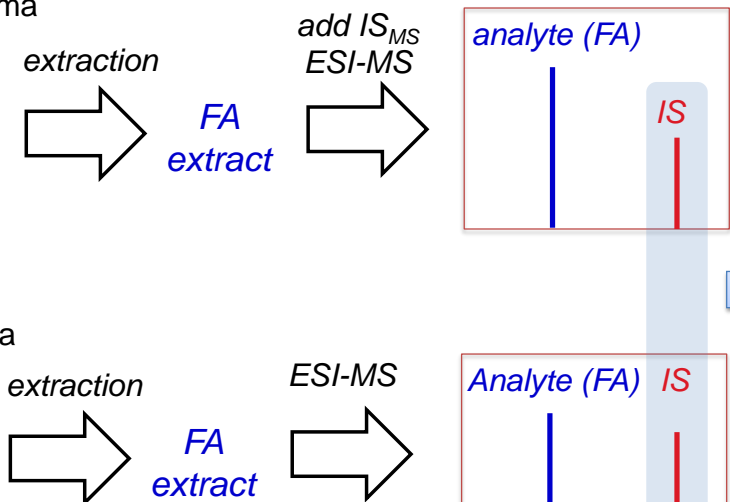

$\sim 80 \%$

recovery

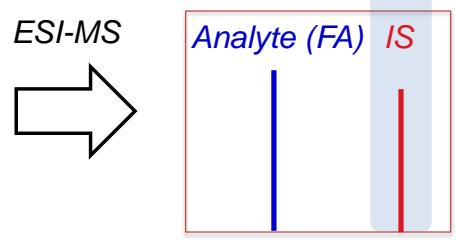

Scheme S2. Principle to estimate the recovery of FAs in human plasma (using a LIPID-MAPS FAs extraction protocol) 


\begin{tabular}{|c|c|c|c|c|}
\hline $\begin{array}{c}\text { stds added before } \\
\text { extraction }\end{array}$ & $\begin{array}{c}\text { ratio } \\
(\mathrm{D} / \mathrm{H})\end{array}$ & $\begin{array}{c}\text { stds added after } \\
\text { extraction }\end{array}$ & $\begin{array}{c}\text { ratio } \\
(\mathrm{D} / \mathrm{H})\end{array}$ & Recovery $(\%)$ \\
\hline $\mathrm{C} 16: 1-\mathrm{d} 14 / \mathrm{C} 16: 1$ & 6.43 & $\mathrm{C} 16: 1-\mathrm{d} 14 / \mathrm{C} 16: 1$ & 8.09 & 79.5 \\
\hline $\mathrm{C} 18: 1-\mathrm{d} 17 / \mathrm{C} 18: 1$ & 0.84 & $\mathrm{C} 18: 1-\mathrm{d} 17 / \mathrm{C} 18: 1$ & 1.05 & 80.2 \\
\hline $\mathrm{C} 18: 2-\mathrm{d} 11 / \mathrm{C} 18: 2$ & 1.27 & $\mathrm{C} 18: 2-\mathrm{d} 11 / \mathrm{C} 18: 2$ & 1.62 & 78.2 \\
\hline $\mathrm{C} 20: 4-\mathrm{d} 8 / \mathrm{C} 20: 4$ & 1.61 & $\mathrm{C} 20: 4-\mathrm{d} 8 / \mathrm{C} 20: 4$ & 2.00 & 80.5 \\
\hline
\end{tabular}

\subsection{Calibration curves for unsaturated FA analysis}

Table S1. Calibration curves, LODs and LOQs for the analysis of unsaturated FAs by PB reaction followed by NLS (58 Da).

\begin{tabular}{|c|c|c|c|c|}
\hline Fatty Acid & Calibration curve & $R^{2}$ & LOD $(\mu \mathrm{M})$ & LOQ $(\mu \mathrm{M})$ \\
\hline $\begin{array}{c}\text { palmitoleic acid } \\
\text { (FA 16:1) }\end{array}$ & $y=0.1007 x+0.0262$ & 0.9972 & 0.039 & 0.39 \\
\hline $\begin{array}{c}\text { linoleic acid } \\
\text { (FA 18:2) }\end{array}$ & $y=0.1116 x+0.0149$ & 0.9948 & 0.071 & 0.36 \\
\hline $\begin{array}{c}\text { oleic acid/cis-vaccenic acid }{ }^{[\mathrm{a}]} \\
\text { (FA 18:1) }\end{array}$ & $y=0.1274 x+0.0324$ & 0.9996 & 0.035 & 0.35 \\
\hline $\begin{array}{c}\text { arachidonic acid } \\
\text { (FA 20:4) }\end{array}$ & $y=0.2774 x-0.0621$ & 0.9961 & 0.33 & 0.66 \\
\hline $\begin{array}{c}\alpha-\text { linolenic acid } \\
\text { (FA 18:3) }\end{array}$ & $y=0.1887 x-0.0416$ & 0.9912 & 0.10 & 0.31 \\
\hline
\end{tabular}

[a] The molar composition of FA 18:1 was pre-determined to be $91.5 \%$ oleic acid and $8.5 \%$ cis-vaccenic acid, and used to make standard solutions to prepare the calibration curve for FA 18:1. Solvent for quantitation of FA 16:1, FA 18:1, and FA 18:2 is $5 \%$ ethanol in acetone/ $\mathrm{H}_{2} \mathrm{O}(50 / 50$, v/v). [b] Solvent for quantitation of FA 18:2, 18:3, and 20:4: ethanol/acetone/water $(40 / 30 / 30, \mathrm{v} / \mathrm{v} / \mathrm{v})$. 


\subsection{Quantitation of low-abundance PUFAs and their isomers}

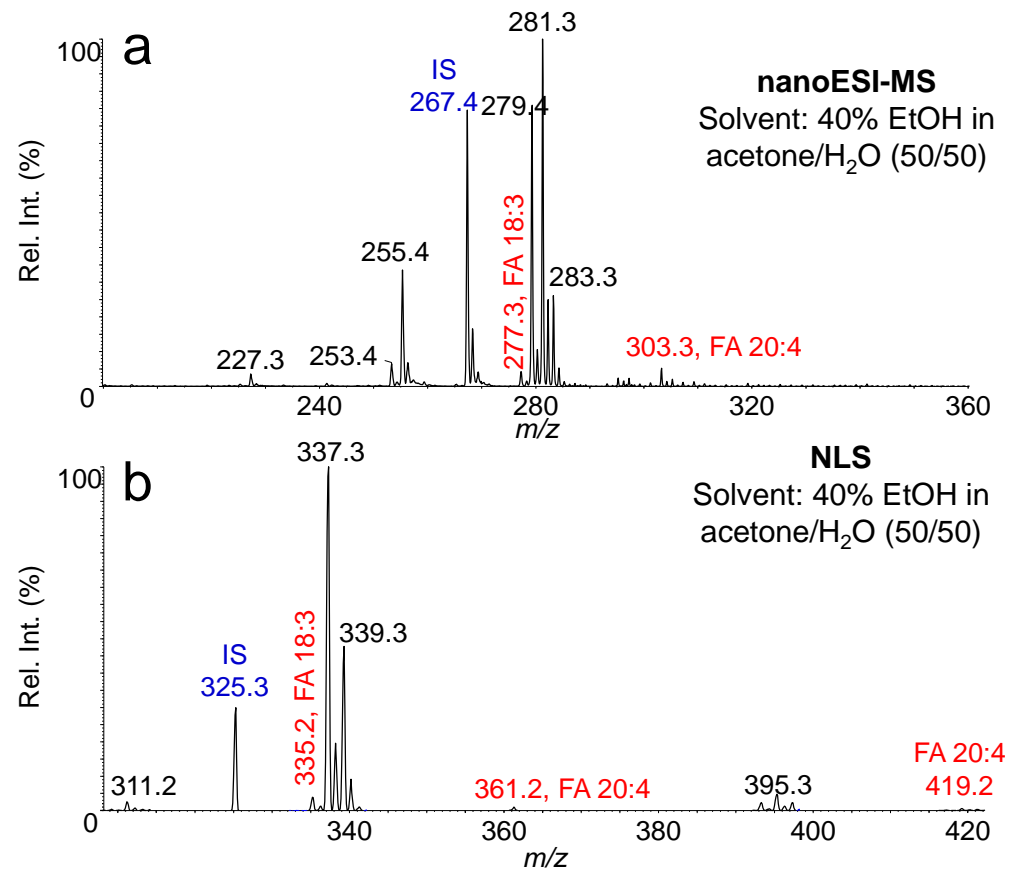

Figure S4. Analysis of PUFAs (FA 18:3 and FA 20:4) in human plasma. (a) NanoESI-MS spectrum of FA extract added with 7.5 $\mu$ M FA 17:1 as the IS. (b) NLS spectrum after PB reaction. Dried FA extract (from $20 \mu \mathrm{L}$ human plasma) was reconstituted in $400 \mu \mathrm{L}$ solvent (ethanol/acetone/water (40/30/30, v/v/v) added with $7.5 \mu \mathrm{M}$ IS.
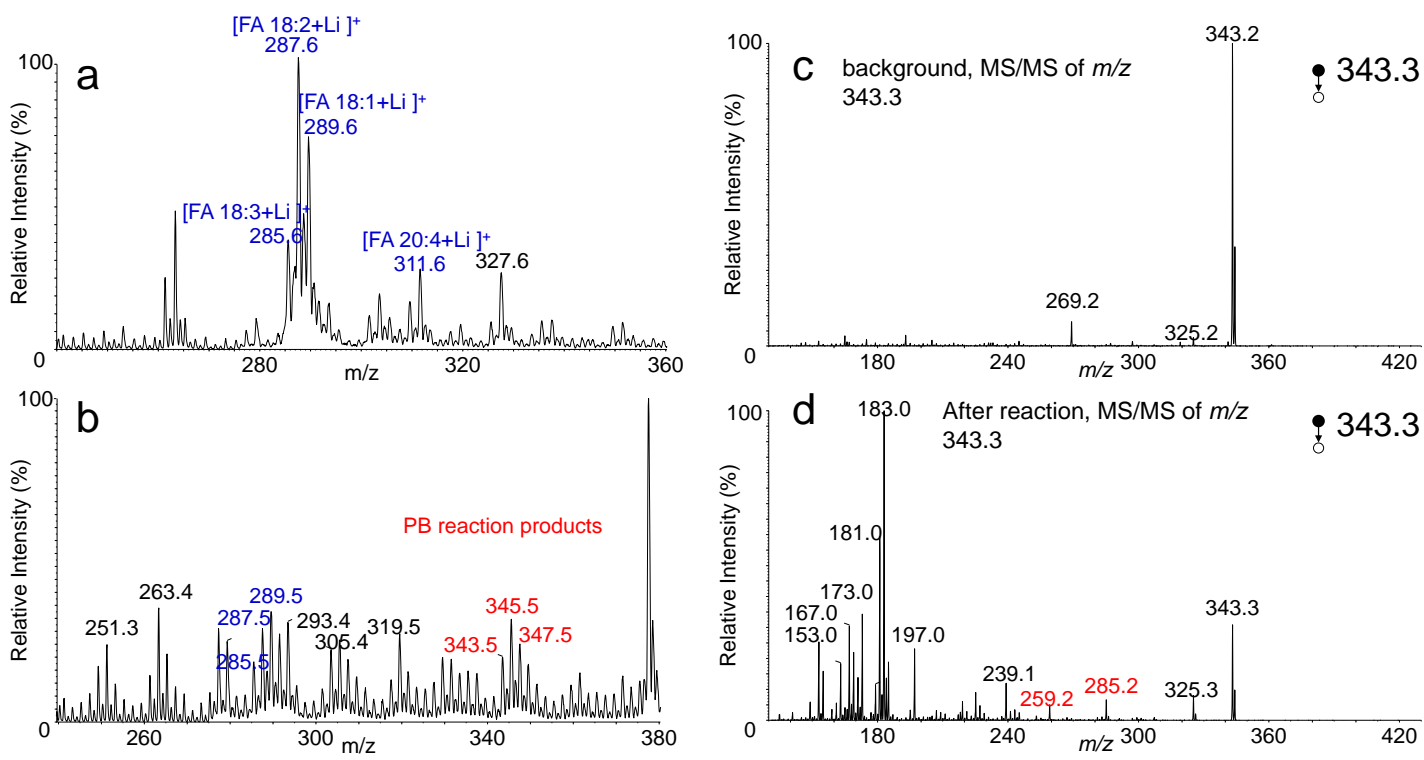

Figure S5. FA 18:3 in human plasma is a mixture of $\omega$-3 (FA 18:3 $(9,12,15), \alpha$-linolenic acid, ALA) and $\omega$-6 (FA 18:3 $(6,9,12), \gamma$-linolenic acid, GLA) isomers. Since FA 18:3 is a PUFA, no abundant diagnostic ions in MS/MS can be produced after tagging, due to the more dominant 44 Da loss (in negative ion mode). We therefore made the analysis of FA 18:3 possible by +nanoESI-MS by adding lithium chloride $(5 \mathrm{mM})$. Abundant diagnostic ions can be produced accordingly, and the 44 Da neutral loss is suppressed. (a) nanoESI-MS spectrum of the FA extract 
after addition of $5 \mathrm{mM} \mathrm{LiCl}$. Solvent is $10 \%$ ethanol in acetone/water $(50 / 50, \mathrm{v} / \mathrm{v})$. (b) nanoESI-MS spectrum of FA extract after photochemical tagging. Peaks labelled in red are tagged products of FA 18:3 (m/z 343.5), FA 18:2 ( $\mathrm{m} / \mathrm{z} 345.5)$, and FA 18:1 ( $\mathrm{m} / \mathrm{z} 347.5)$. (c) CID spectrum of the background ions at $m / z$ 343. 3. (d) CID spectrum of tagged FA 18:3 ( $m / z$ 343.3).

\subsection{Cross-validation by charged-switched AMPP derivatization of FAs.}

AMPP derivatization has been developed as an effective method to improve the detection sensitivity for fatty acids. Here in this study, we employed this strategy for the quantitation of fatty acids in human plasma to validate the developed PB/NLS method, using linoleic acid-d11 and oleic acid- $d 17$ as the internal standards. The isotopologue has the same ionization efficiency as the corresponding fatty acid. After derivatization, all fatty acids and internal standards can be detected with high sensitivity by positive nanoESI-MS, and each AMPP-derivatized fatty acid produce a characteristic ion at $\mathrm{m} / z 183.3$ upon CID (Figure S6). By comparing the peak ratio between the fatty acid and its corresponding isotopologue in the precursor ion scan (PIS) spectrum, the concentrations of FA 18:1 and FA 18:2 in human plasma were determined to be $254 \mu \mathrm{M}$ and 180 $\mu \mathrm{M}$, consistent with the results obtained by the PB/NLS method $(276 \pm 36 \mu \mathrm{M}$ and $193 \pm 20 \mu \mathrm{M})$.

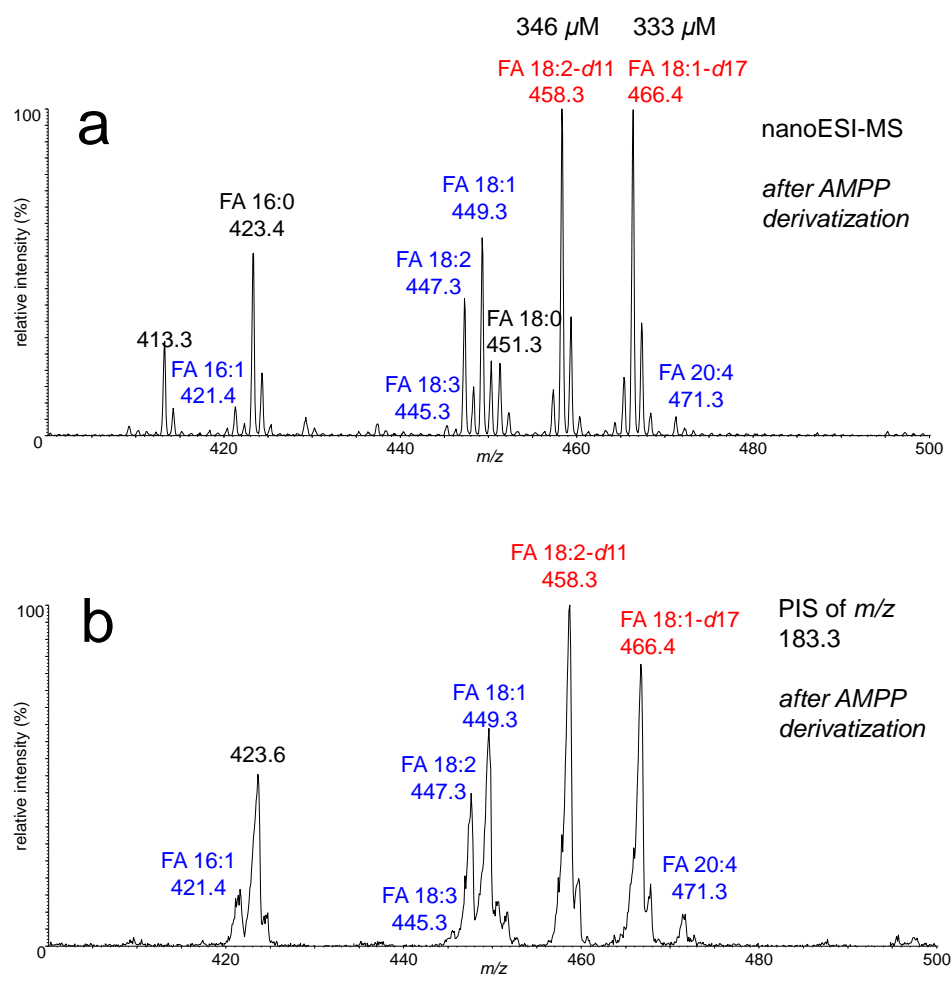

Figure S6. Quantitation of unsaturated FAs in human plasma using the AMPP method. ${ }^{3}$ (a) nanoESI-MS spectrum of the FA extract of human plasma after AMPP derivatization. (b) Precursor ion scan spectrum of $\mathrm{m} / \mathrm{z} 183.1$ of the same extract after AMPP derivatization. FA 18:2- $d 11$ and FA 18:1- $d 17$ were added before extraction to evaluate recovery. 


\section{Analysis of unsaturated FAs in normal and cancerous prostate cells.}
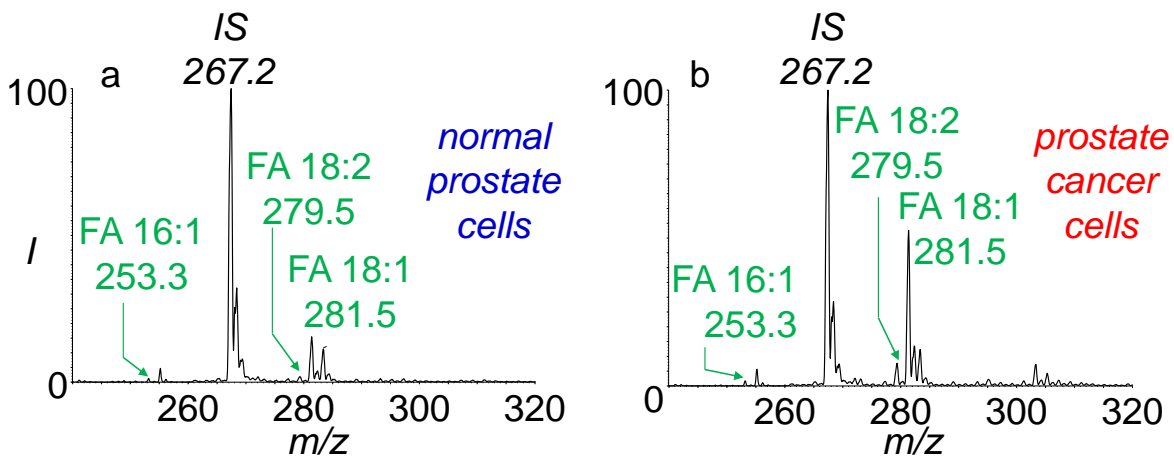

Figure S7. FA profiles of normal and cancerous prostate cells. (a) nanoESI-MS spectrum of FAs in normal prostate cells (RWPE1 cells). (b) nanoESI-MS spectrum of FAs in cancerous prostate cells (PC3 cells). FA 17:1 (10Z) was used as the IS.
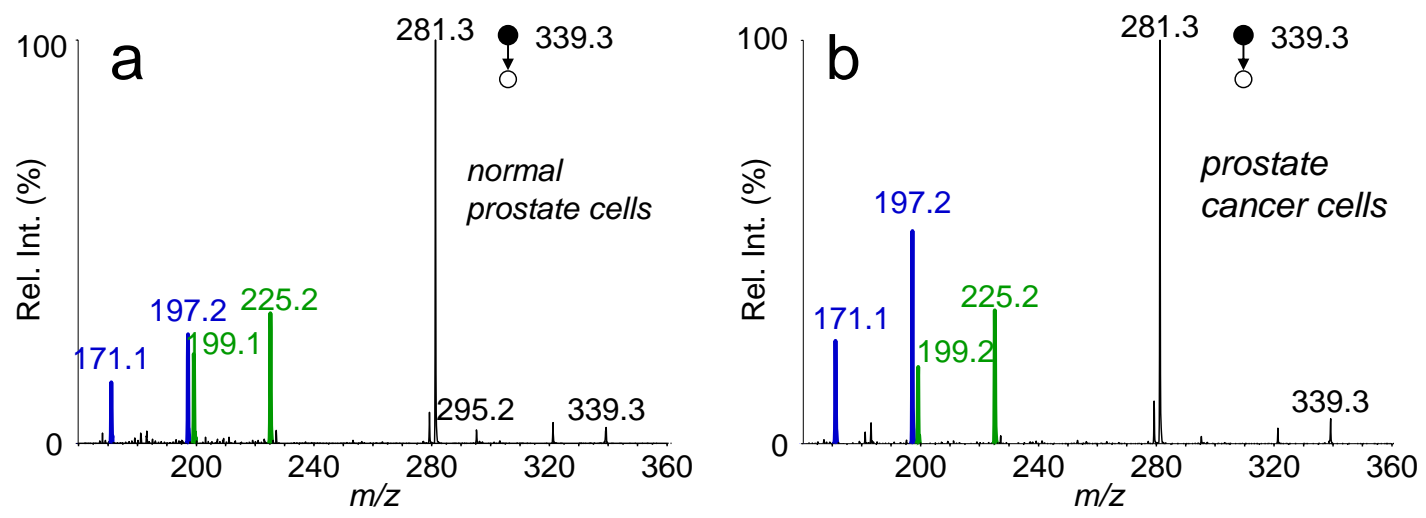

Figure S8. FA 18:1 in normal and cancerous human prostate cells is a mixture of $9 \mathrm{Z}$ and $11 \mathrm{Z}$ isomers. (a) Trap CID spectrum of PB reaction products $(\mathrm{m} / \mathrm{z}$ 339.3) of FA 18:1 in normal prostate cells. (b) Trap CID spectrum of PB reaction products of FA 18:1 in prostate cancer cells. Trap CID conditions: Q3 entry barrier, 2V; activation time, $200 \mathrm{~ms}$; AF2 (fragmentation energy), 50 (arbitrary unit). Diagnostic ions at $m / z$ 171.1/197.2 are unique to the $\Delta 9$ isomer, while diagnostic ions at $\mathrm{m} / \mathrm{z} 199.2 / 225.2$ are unique to $\Delta 11$ isomer.

\section{References:}

1. Ma, X. \& Xia, Y. Pinpointing double bonds in lipids by Paternò-Büchi reactions and mass spectrometry. Angew. Chem. Int. Ed. 53, 2592-2596 (2014).

2. Ma, X. et al. Identification and quantitation of lipid $\mathrm{C}=\mathrm{C}$ location isomers: A shotgun lipidomics approach enabled by photochemical reaction. Proc. Natl. Acad. Sci. U.S.A. 113, 2573-2578 (2016).

3. Yang, K., Dilthey, B.G. \& Gross, R.W. Identification and Quantitation of Fatty Acid Double Bond Positional Isomers: A Shotgun Lipidomics Approach Using Charge-Switch Derivatization. Anal. Chem. 85, 9742-9750 (2013). 